\title{
Lista revisada y clave para los corales pétreos zooxantelados (Hydrozoa: Milleporina; Anthozoa: Scleractinia) del Atlántico mexicano
}

\author{
Aurora U. Beltrán-Torres \& Juan P. Carricart-Ganivet \\ Departamento de Ecología Acuática, ECOSUR. Apdo. Postal 424. Chetumal, Q. Roo. 77000. México. Fax (983) \\ 20447. Correo electrónico: abeltran @ecosur-qroo.mx, jpcarri@ecosur-qroo.mx
}

Recibido 4-VIII-1998. Corregido 23-IV-1999. Aceptado 11-V-1999

\begin{abstract}
A revised and annotated checklist of fifty-seven zooxanthellate stony coral species (three milleporids and fifty-four scleractinian) is presented for the Mexican East Coast. This report includes synonyms and a simplified key to species on the list. Morphological and taxonomic observations are included when authors disagree in the taxonomic position and/or identification of the species, as well as when taxonomic information is pertinent, or when distribution data are relevant for some species in the Mexican Atlantic. A qualitative analysis of species abundance and geographic distribution, and bathymetric range data are presented. There is a reduction in the genera and species numbers from the Mexican Caribbean to the Gulf of Mexico and there, from the Campeche Bank to Veracruz. Furthermore, the number of abundant species is lower in the Gulf of Mexico than in the Mexican Caribbean, and the common species for all reef zones have a wider bathymetric range in the Mexican Caribbean.
\end{abstract}

Key words: Coral reefs, diversity, Gulf of Mexico, Mexican Caribbean, Campeche Bank, Veracruz.

Las aguas del litoral oriental mexicano se pueden dividir en dos grandes zonas: la del Golfo de México y la del Caribe. En el Golfo de México existen formaciones arrecifales que se pueden diferenciar en tres regiones: 1) Veracruz Norte, al sudeste de Cabo Rojo, frente a la laguna de Tamiahua y frente a Tuxpan, 2) Veracruz Sur, representado por el Sistema Arrecifal Veracruzano (SAV), frente al Puerto de Veracruz y el poblado de Antón Lizardo y 3) Banco de Campeche, considerado como una extensión sumergida de la Península de Yucatán de $200 \mathrm{~km}$ hacía el oeste y noroeste. Por otro lado, las costas orientales de la Península de Yucatán están bordeadas por una extensa formación arrecifal, en algunos lugares más desarrollada que en otros (Carricart-Ganivet y HortaPuga 1993).

Horta-Puga y Carricart-Ganivet (1993), en un listado de corales pétreos recientes de Mé- xico, revisaron toda la literatura publicada y registraron para el Atlántico una especie de mileporino (con tres formas, consideradas aquí como especies separadas), doce de estilasterinos, de las que sólo una habita en los arrecifes, y noventa y siete de escleractinios, de las cuales cuarenta y seis son zooxanteladas. En el presente trabajo se listan tres especies de mileporinos y cincuenta y cuatro de escleractinios, incrementado el número de corales pétreos zooxantelados conocidos para aguas someras del Atlántico mexicano a cincuenta y siete, siendo este el listado más grande de especies publicado hasta el momento para la zona. Además, se incluye una clave dicotómica simplificada para la determinación de dichas especies.

Los datos que forman la base de esta lista revisada y con los que se elaboró la clave de determinación fueron tomados de la literatura (Heilprin 1890, Moore 1958, Kornicker et al. 
1959, Villalobos 1971, Jordán 1979, 1980, 1988, 1990 y 1993, Castañares y Soto 1982, Farrel et al. 1983, Chávez e Hidalgo 1984, Jordán y Martín 1987, Fenner 1988, 1993a y 1999, Tunnell 1988, Reyes-Castro et al. 1989, Carricart-Ganivet y Horta-Puga 1990, Muckelbauer 1990, Lara et al. 1992, Padilla et al. 1992, Tunnell et al. 1993, Carricart-Ganivet y Beltrán-Torres 1997), así como del material depositado en la colección de ECOSUR. Las categorías supreaespecíficas y la secuencia filogenética de las familias que aparecen en la lista siguen a Boschma (1956) para mileporinos y a Verón (1995) para escleractinios.

Las especies se acomodan alfabéticamente dentro de los géneros. Debido a la incoherencia en la literatura, se incluyen las referencias de los trabajos en donde se ha descrito a la especie o con fotografías de ella; asimismo, se mencionan las sinonimias de aquellas especies que han sido registradas con otro nombre en la zona. En algunos casos, se incluyen observaciones sobre morfología o taxonomía.

En el Cuadro 1 se da la abundancia relativa para las localidades estudiadas, para lo cual se usan cuatro categorías: a) abundante.- se puede encontrar en todas o en casi todas las localidades estudiadas o en alguna de las localidades se le cita como una especie conspicua de la misma, b) común.- frecuentemente registrada, aunque no en todas las localidades o en toda ocasión; c) ocasional.- se registra sólo en alguna de las localidades estudiadas y se le cita como observada sólo en algunas ocasiones y 4) rara.- especie registrada excepcionalmente. En el Cuadro 2 se mencionan las localidades del Atlántico y Caribe mexicanos en donde se ha registrado a la especie incluyendo, en caso de estar disponible, el ámbito de profundidad para cada una. En ambos Cuadros, la información se obtuvo de la bibliografía mencionada en el párrafo superior y se complementó con observaciones personales.

En términos del número de géneros y especies, es evidente una reducción del Caribe mexicano al Golfo de México y, dentro de este, del Banco de Campeche a Veracruz. Además, de manera general puede decirse que el número de especies abundantes o comunes es menor en el Golfo de México que en el Caribe (Cuadro 1). Asimismo, las especies comunes a las tres zonas, presentan una distribución batimétrica más amplia en el Caribe que en el Golfo de México (Cuadro 2). Por un lado, esto puede ser reflejo de las limitaciones de los muestreos en términos espaciales y temporales; por ejemplo, un mayor esfuerzo de muestreo en arrecifes más cercanos a la costa y, por lo tanto, más accesibles (Jordán 1993). Por otro lado, puede ser reflejo del menor desarrollo arrecifal que se da en el Golfo de México, producto de las condiciones ambientales prevalecientes en este (Carricart-Ganivet y HortaPuga 1993).

\section{Lista de especies Phylum Cnidaria Classis Hydrozoa Ordo Milleporina Familia Milleporidae}

Millepora alcicornis Linnaeus, 1758

Millepora alcicornis.- Squires 1958: 259, Lám. 43: Figs. 1-2.- Duarte Bello 1961: 82, Figs. 71-72.- Almy y Carrión-Torres 1963: 144, Lám. 2: Fig. a.- Olivares y Leonard 1971: 58, Lám. 5: Figs. c-d.- Smith 1971: 95-96.Roos 1971: 43-44, Lám. 2.- Chassaing et al. 1978: 61,63, Figs. 5-6.- Cairns 1982: 272, Fig. 119a.- Weerdt 1984: 248, 250, Lám. 1: Figs. 13, Lám. 5: Figs. 1-2.

\section{Millepora complanata Lamarck, 1816}

Millepora squarrosa.- Roos 1971: 44, Lám. 1.

Millepora complanata.- Squires 1958: 259, Lám. 43: Fig. 3.- Duarte Bello 1961: 84, Figs. 73-74.- Almy y Carrión-Torres 1963: 144, Lám. 2: Fig. b.- Roos 1971: 44-45, Lám. 3.- Chassaing et al. 1978:63, Figs. 7-8.- Cairns 1982: 272-273, Fig. 119b-c.- Weerdt 1984: 250, 255, Lám. 2: Figs. 1-4, Lám. 5: Figs. 3-4, Lám. 7: Fig. 1.

Millepora squarrosa Lamarck, 1816 Millepora squarrosa.- Almy y Carrión- 
Torres 1963: 144, Lám. 3: Fig. a.- Olivares y Leonard 1971: 61, Lám. 6: Figs. a-b.- Chassaing et al. 1978: 63, Fig. 9.- Weerdt 1984: 252, Lám. 3: Figs. 1-3, Lám. 6: Figs. 1-2.Weerdt 1990: Lám. 1, Figs. 1-6.

Observaciones: Se ha afirmado que esta especie tiene una distribución discontinua en el Caribe, restringida a la República Dominicana, Puerto Rico, Las Antillas Menores y Brasil (Weerdt 1990), y que los registros de esta especie en el Caribe Noroccidental y en el Golfo de México son erróneos (Fenner com. pers.). Se encuentra incluida en este trabajo ya que uno de nosotros (JPCG) observó especímenes, en el arrecife de Triángulo Oeste, que se ajustaban a la descripción dada por Weerdt (1984) para la especie. Sin embargo, es necesario recolectar algunos ejemplares para confirmar su identificación.

\section{Classis Anthozoa \\ Subclassis Zoantharia \\ Ordo Scleractinia \\ Subordo Archaecoeniina \\ Familia Astrocoeniidae}

Stephanocoenia intersepta (Esper, 1795)

Stephanocoenia michelinii.- Squires 1958:

246, Lám. 32: Figs. 1-2.- Duarte Bello 1961: 80, Figs. 69-70.- Almy y Carrión-Torres 1963: 144, Lám. 3: Fig. b.- Olivares y Leonard 1971: 67, Lám. 11: Figs. a-b.- Smith 1971: 72, Lám. 2.- Chassaing et al. 1978: 63, Figs. 11-12.Castañares y Soto 1982: 304, Lám. 1: Figs. ab.- Cairns 1982: 274, Fig. 120a, b.- Cortés y Guzmán 1985: 66, Fig. 1a.

Stephanocoenia intersepta.- Roos 1971: 51, Lám. 4: Fig. c, Lám. 5: Figs. a-b.

\section{Familia Pocilloporidae}

Madracis decactis (Lyman, 1859)

Madracis decactis.- Duarte Bello 1961: 46, Figs. 35-36.- Smith 1971: 72, Lám. 1.Wells 1973: 19 (clave de identificación).- Roos 1971: 52-53, Lám. 8: Figs. a-b.- Chassaing et al. 1978: 63, Fig. 13.-Castañares y Soto 1982: 304, Lám. 1: Figs. c-d.- Cairns 1982: 274, Fig. 120c-d.- Cortés y Guzmán 1985: 66, Fig. 1b.
Madracis mirabilis (Duchassaing \& Michelotti, 1860)

Madracis asperula.- Roos 1971: 59, Lám. 9: Fig. b, Lám. 10.

Madracis mirabilis.- Wells 1973: 19 (clave de identificación).- Chassaing et al. 1978: 63, Fig. 14.- Cairns 1982: 274, 276, Fig. 120ef.- Cortés y Guzmán 1985: 66-67, Fig. 2a-b.

Familia Acroporidae

Acropora cervicornis (Lamarck, 1816)

Acropora cervicornis.- Duarte Bello 1961: 12. Almy y Carrión-Torres 1963: 145-146, Lám. 4: Fig. a.- Roos 1971: 54-55, Lám. 9: Fig. a, Lám. 12: Fig. a.- Smith 1971: 73, Lám. 3.- Chassaing et al. 1978: 63, Fig. 15.- Castañares y Soto 1982: 305, Lám. 2: Figs. a-b.Cairns 1982: 276, Fig. 121d.- Cortés y Guzmán 1985: 67, Fig. 3a-b.

Acropora palmata (Lamarck, 1816)

Acropora palmata.- Squires 1958: 246247, Lám. 34: Fig. 1.- Duarte Bello 1961: 14, Figs. 3-4.- Almy y Carrión-Torres 1963: 146, Lám. 4: Fig. b.- Olivares y Leonard 1971: 53, Lám. 1.- Roos 1971: 55-56, Lám. 12: Fig. b.Smith 1971: 73-74, Lám. 4.- Chassaing et al. 1978: 63, Figs. 16-17.- Castañares y Soto 1982: 305, Lám. 2: Figs. c-d.- Cairns 1982: 276, Fig. 120b, c.- Cortés y Guzmán 1985: 67, Fig. 4a-b.

Acropora prolifera (Lamarck, 1816)

Acropora prolifera.- Duarte Bello 1961: 1213, Figs. 1-2.- Almy y Carrión-Torres 1963: 146, Lám. 5: Fig. a.- Roos 1971: 55, Lám. 11.- Smith 1971: 74.- Castañares y Soto 1982: 306, Lám. 3: Figs. a-b.- Cairns 1982: 276, 279, Fig. 121e.

\section{Subordo Poritiina} Familia Poritidae

\section{Porites astreoides Lamarck, 1816}

Porites astreoides.- Squires 1958: 250-251, Lám. 39: Figs. 2-3.- Duarte Bello 1961: 64, Figs. 53-54.- Almy y Carrión-Torres 1963: 149, Lám. 8: Fig. a. - Roos 1971: 61-62, Lám. 18: Fig. a y Lám. 19: Fig. a.- Olivares y Leonard 
1971: 64, Lám. 9: Figs. a-b.- Smith 1971: 77, Lám. 15 (no-Lám. 19).- Chassaing et al. 1978: 69, Figs. 30-31.- Castañares y Soto 1982: 310, Lám. 9: Figs. a-b.- Cairns 1982: 281-282, Fig. 124d.- Cortés y Guzmán 1985: 70, Fig. 15a-b.

\section{Porites branneri Rathbun, 1888}

Porites branneri.- Roos 1971: 60, Lám. 18: Fig. b; Lám. 19: Fig. b.- Smith 1971: $77-$ 78, Lám. 4.- Chassaing et al. 1978: 69.

Observaciones: No hay seguridad de que los organismos registrados como $P$. branneri en el Caribe correspondan a aquella especie descrita como endémica de Brasil (Weil 1992).

Porites colonensis Zlatarski, 1990

Porites colonesis.- Zlatarski 1990: 257268, Figs. 1-20.

\section{Porites divaricata Lesueur, 1821}

Porites porites var. divaricata.- Squires 1958: 252-253, Lám. 38: Fig. 3.- Duarte Bello 1961: 70, Figs. 59-60.- Almy y Camión-Torres 1963: 149-150, Lám. 8 Fig. b. - Roos 1971: 59 (clave de identificación).- Cairns 1982: 283, Fig. 125a.- Cortés y Guzmán 1985: 70-71, Fig. 17b.

Porites divaricata -Smith 1971: 78.Chassaing et al. 1978: 69, Fig. 29 (a la derecha).- Castañares y Soto 1982: 311, Lám. 10: Figs. c-d.

\section{Porites furcata Lamarck, 1816}

Porites porites var. furcata.- Squires 1958: 252, Lám. 39: Fig. 1.- Duarte Bello 1961: 68, Figs. 57-58.- Almy y Carrión-Torres 1963: 149, Lám. 9 Fig. b. - Roos 1971: 59 (clave de identificación).- Cairns 1982: 283, Fig. 125c.- Cortés y Guzmán 1985: 70-71, Fig. 17a.

Porites furcata.- Smith 1971: 78, Lám. 12.- Chassaing et al. 1978: 69, Fig. 29 (a la izquierda).- Castañares y Soto 1982: 310-311, Lám. 10: Figs. a-b.

\section{Porites porites (Pallas, 1766)}

Porites porites var. clavaria.- Squires 1958: 251-252, Lám. 38: Figs. 1-2.- Duarte Bello 1961: 66, Figs. 55-56.- Almy y CarriónTorres 1963: 149, Lám. 9: Fig. a. - Roos 1971:
59-60, Lám. 16: Figs. a-b y Lám. 17: Figs. ab.- Cortés y Guzmán 1985: 70-71, Fig. 16a-b.

Porites porites.- Olivares y Leonard 1971: 64, Lám. 9: Figs. c-d.- Smith 1971: 78-79, Láms. 13, 14.- Chassaing et al. 1978: 69, Fig. 28.- Castañares y Soto 1982: 310, Lám. 9: Figs. c-d.- Cairns 1982: 283 Fig. 124e-f, 125b.

Observaciones: En el Caribe, tradicionalmente ha existido un gran desacuerdo en cuanto a que si Porites porites, $P$. divaricata y $P$. furcata son especies diferentes o simples formas de la misma especie (Cairns 1982). Aquí las tratamos como especies diferentes con base en el trabajo de Weil (1992), en el que hizo un estudio morfométrico multivariado en combinación con técnicas moleculares, concluyendo que se trata de especies separadas.

\section{Subordo Fungiina \\ Familia Siderastreidae}

\section{Siderastrea radians (Pallas, 1766)}

Siderastrea radians.- Squires 1958: 248249, Lám. 35: Figs. 1-4, Lám. 36: Fig. 3.Duarte Bello 1961: 72, Figs. 61-62.- Almy y Carrión-Torres 1963: 148, Lám. 7: Fig. b. Olivares y Leonard 1971: 67, Lám. 10: Figs. ab.- Roos 1971: 62, Láms. 20-21.- Smith 1971: 76, Lám. 15.- Chassaing et al. 1978: 66, Fig. 26.- Castañares y Soto 1982: 309, Lám. 8: Figs. a-b.- Cairns 1982: 281, Fig. 124a-b.Cortés y Guzmán 1985: 69, Fig. 13a-b.

Observaciones: Esta especie ha sido registrada tanto en el Océano Índico como en el Atlántico, si la identificación de los registros del Indico es correcta, entonces es la única especie que se presenta en ambos océanos (Veron 1995).

Siderastrea siderea (Ellis \& Solander, 1786)

Siderastrea siderea.- Squires 1958: 249250, Lám. 36: Figs. 1-2.- Duarte Bello 1961: 74, Figs. 63-64.- Almy y Carrión-Torres 1963 : 148, Lám. 7: Fig. c. - Olivares y Leonard 1971: 67, Lám. 10: Figs. c-d.- Roos 1971: 63-64, Lám. 13: Figs. a-b.- Smith 1971: 76-77, Lám. 10.- Chassaing et al. 1978: 67, Figs. 25 27.Castañares y Soto, 1982: 309-310, Lám. 8: 
Figs. c-d.- Cairns 1982: 281, Fig. 124c.- Cortés y Guzmán 1985: 70, Fig. 14a-b.

Observaciones: En el Golfo de México existe una intergradación de caracteres entre Siderastrea radians y $S$. siderea (Carricart-Ganivet y Beltrán-Torres 1997), por lo que las dos especies no pueden ser separadas de manera clara. Para el Caribe, Fenner (1993b) hizo un estudio morfológico con ambas especies en varias localidades, incluyendo a México (Cozumel), en el que concluyó que ambas representan especies validadas.

\section{Familia Agariciidae}

Agaricia agaricites (Linnaeus, 1758)

Agaricia agaricites.- Squires 1958: 247, Lám. 33: Figs. 1-2.- Duarte Bello 1961: 16-21 Figs. 5-10.- Almy y Carrión-Torres 1963: 146147, Lám. 5: Fig. b, Lám. 6: Fig. a. - Olivares y Leonard 1971: 53, Lám. 1: Figs. c-d.- Roos 1971: 55-58, Lám. 14: Figs. a-b.- Smith 1971: 74-75, Láms. 5-6.- Wells 1973: 25, Fig. 6a (no A. fragilis).- Chassaing et al. 1978: 66, Figs. 18-19.- Castañares y Soto 1982: 306-307, Lám. 3: Figs. c-d, Lám. 4: Figs. a-b, Lám. 5: Figs. a-c.- Cairns 1982: 279, Fig. 122b-e.- Cortés y Guzmán 1985: 68, Figs. 5-9.

Observaciones: Se han descrito cinco formas para esta especie, las formas registradas en la zona de estudio son: agaricites, es la más abundante y se registra en todas las localidades entre los 3 y $20 \mathrm{~m}$ de profundidad, purpurea (2-20 m), también se encuentra en todas las localidades aunque puede considerarse menos abundante que la anterior, las otras tres formas, carinata, humilis y danai sólo han sido registradas en el Caribe mexicano, en donde se encuentran de manera abundante a común.

\section{Agaricia fragilis (Dana, 1848)}

Agaricia fragilis.- Duarte Bello 1961: 2223, Figs. 11-12.- Almy y Carrión-Torres 1963: 147, Lám. 6: Fig. b..- Smith 1971: 75, Lám. 8.Wells 1973: 24 Figs. 6b-7 (no-Fig. 6a = A. agaricites).- Chassaing et al. 1978: 66, Fig. 20.Castañares y Soto 1982: 308, Lám. 7: Figs. ab.- Cairns 1982: 279, Fig. 123a-b.- Cortés y Guzmán 1985: 68-69, Fig. 10a-b.
Agaricia grahamae Wells, 1973

Agaricia grahamae.- Wells 1973: 28-29, 31, Figs. 5, 11, 12a-b.- Chassaing et al. 1978: 66, Fig. 22.

Agaricia lamarcki (Milne Edwards \& Haime, 1851)

Agaricia lamarcki.- Wells 1973: 26-28, Figs. 8-10.- Chassaing et al. 1978: 66, Fig. 21.Castañares y Soto 1982: 307-308, Lám. 6: Figs. c-d.- Cairns 1982: 281, Fig. 123e-f.

Agaricia tenuifolia Dana, 1848

Agaricia tenuifolia.- Wells 1973: 25 (clave de identificación), Fig. 13.- Castañares y Soto 1982: 307, Lám. 6: Figs. a-b. Cairns 1982: 279, Fig. 122f.- Cortés y Guzmán 1985: 69, Fig. 11a-b.

Agaricia undata (Ellis \& Solander, 1786)

Agaricia undata.- Wells 1973: 24, Figs. 4-

5.- Chassaing et al. 1978: 66, Fig. 23.

Leptoseris cucullata (Ellis \& Solander, 1786) Agaricia nobilis.- Duarte Bello 1961: 22.Smith 1971: 75, Lám. 7.

Agaricia agaricites.- Roos 1971: 56-58, Lám. 15.

Agaricia cucullata.- Almy y Carrión-Torres 1963: 147, Lám. 7: Fig. a.

Helioseris cucullata.- Wells 1973: 25, Figs. 14a-b, 33.- Chassaing et al. 1978: 66, Fig. 24.Castañares y Soto 1982:308-308, Lám. 7: Figs. c-d.- Cortés y Guzmán 1985: 69, Fig. 12a-b.

Leptoseris cucullata.- Cairns 1982: 281, Fig. 123c-d.

\section{Familia Oculinidae}

\section{Subordo Meandriina}

\section{Oculina diffusa Lamarck, 1816}

Oculina difussa.- Squires 1958: 256-257, Lám. 38: Fig. 4.- Duarte Bello 1961: 60-61 Figs. 49-50.- Almy y Carrión-Torres 1963: 156, Lám. 16: Fig. a.- Olivares y Leonard 1971: 64, Lám. 8: Figs. a-b.- Smith 1971: 88, Lám. 34.- Chassaing et al. 1978: 74, Fig. 51.Cortés y Guzmán 1985: 75-76, Fig. 29a-b. 
Oculina valenciennesi Milne Edwards \& Haime, 1848

Oculina valenciennesi.- Duarte Bello 1961: 62.- Roos 1971: 75, Lám. 37.- Smith 1971: 88.- Chassaing et al. 1978: 74, Fig. 52.Reyes-Castro et al. 1989: 7, Fig. 4.

\section{Familia Meandrinidae}

Meandrina meandrites (Linnaeus, 1758)

Meandrina meandrites.- Duarte Bello 1961: 50 Figs. 39-40.- Almy y Carrión-Torres 1963: 156-157, Lám. 16: Fig. b.- Olivares y Leonard 1971: 59, Lám. 5: Figs. a-b.- Roos 1971: 76, Lám. 40: Figs. a-b.- Smith 1971: 89, Láms. 35-36.- Chassaing et al. 1978: 74, 76, Figs. 53-55.- Castañares y Soto 1982: 315, Lám. 16: Figs. c-d, Lám. 17: Figs. a-b.- Cairns, 1982: 290, Fig. 129a-c.- Carricart-Ganivet y Horta-Puga 1990: 27-28, 2 Figs.

Meandrina brasiliensis.- Smith 1971: 8990, Lám. 36.

Observaciones: Esta especie ocurre en tres formas: meandrites, brasiliensis y danae (Cairns 1982), consideradas como especies diferentes por Smith (1971). En el Atlántico mexicano sólo las dos primeras formas han sido registradas; la forma meandrites en los arrecifes del Banco de Campeche y del Caribe (Castañares y Soto 1982, Carricart-Ganivet y Beltrán-Torres 1997), mientras que la forma brasiliensis sólo en una localidad del Caribe mexicano (Carricart-Ganivet y HortaPuga 1990).

\section{Dichocoenia stokesi Milne Edwards \& Haime, 1848}

Dichocoenia stokesi.- Squires 1958: 257, Lám. 34: Fig. 4.- Duarte Bello 1961: 30 Figs. 19-20.- Almy y Carrión-Torres 1963: 157, Lám. 17: Fig. a.- Olivares y Leonard 1971: 56, Lám. 2: Figs. c-d.- Roos 1971: 77-78, Láms. 41-43.- Smith 1971: 90, Lám. 37.Wells 1973: 44-45, Figs. 29-30.- Chassaing et al. 1978: 76, Fig. 56.- Castañares y Soto 1982: 315-316, Lám. 17: Figs. c-d.- Cairns 1982: 292, Fig. 129d-g.- Cortés y Guzmán 1985: 76, Fig. 30a-b.
Dichocoenia stellaris.- Wells 1973: 45-46, Figs. 31-33.- Castañares y Soto 1982: 316, Lám. 18: Fig. a.

Observaciones: Dichocoenia stellaris Milne Edwards \& Haime, 1848 ha sido considerada como una especie diferente con base en su forma de crecimiento más plana y corta y sus cálices no meandroides, en este trabajo se considera una sola especie de acuerdo a las observaciones de Cairns (1982).

\section{Dendrogyra cylindrus Ehrenberg, 1834}

Dendrogyra cylindrus.- Duarte Bello 1961: 28-29, Figs. 17-18.- Almy y Carrión-Torres 1963: 157, Lám. 17: Fig. b.- Roos 1971: 76-77, Láms. 33-34.- Smith 1971: 90-91, Láms. 38-39.- Chassaing et al. 1978: 76, Fig. 57.- Castañares y Soto 1982: 316, Lám. 18: Figs. c-d.- Cairns 1982: 292, Fig. 130a-b.

\section{Subordo Faviina \\ Familia Mussidae}

Scolymia cubensis (Milne Edwards \& Haime, 1849a)

Scolymia cubensis.- Wells 1971: Figs. 1der., 3, 5, 7.- Chassaing et al. 1978: 77, Fig. 59.- Cairns 1982: 294, Fig. 130e-f.

\section{Scolymia lacera (Pallas, 1766)}

Scolymia lacera.- Roos 197-1: 78-79, Láms. 44, Figs. a-b.- Smith 1971: 92-93, Lám. 41.- Wells 1971: Figs. 1-izq., 2, 4, 6.- Chassaing et al. 1978: 77.- Cairns 1982: 294, Fig. 130e-f.

Mussa angulosa forma lacera.- Fenner 1993b: 1105-1106, Lám. 1: Figs. c-d.

Mussa angulosa (Pallas, 1766)

Mussa angulosa.- Duarte Bello 1961: 5657, Figs. 45-46.- Almy y Carrión-Torres 1963: 158, Lám. 18:

Fig. a.- Olivares y Leonard 1971: 61, Lám. 7: Figs. c-d.- Roos 1971: 79-80, Láms. 45-46.Smith 1971: 92, Lám. 42 (no-Lám. 41= Scolymia lacera).- Chassaing et al. 1978: 77, Fig. 58.- Castañares y Soto 1982: 317, Lám. 19: Figs. a-b.- Cairns 1982: 292, Fig. 130c-d.- Fenner 1993b: 1108-1109, Lám. 1: Fig. e. 
Isophyllia multiflora Verril, 1902

Isophyllia multiflora.- Duarte Bello 1961: 44, Figs. 33-34.- Almy y Carrión-Torres 1963: 160, Lám. 19: Fig. b.- Smith 1971: 94-95, Lám. 47.- Chassaing et al. 1978: 77, 79 Fig. 63.

Observaciones: Cairns (1982) consideró a Isophyllia multiflora e I. sinuosa como una misma especie, argumentando que las diferencias entre ambas caen dentro del ámbito de variación de $I$. sinuosa. En los ejemplares que nosotros hemos recolectado en el Caribe mexicano se distinguen perfectamente las diferencias entre ambas especies.

Isophyllia sinuosa.- Castañares y Soto 1982: 317.

Isophyllia sinuosa (Ellis \& Solander, 1786)

Isophyllia sinuosa.- Squires 1958: 2572580, Lám. 40: Fig. 4.- Duarte Bello 1961: 44.Almy y Carrión-Torres 1963: 159-160, Lám. 20: Figs. a-b.- Roos 1971: 81, Láms. 48 y 50.- Smith 1971: 94, Lám. 46.- Chassaing et al. 1978: 77, 79 Fig. 62.- Castañares y Soto 1982: 317, Lám. 19: Figs. c-d.- Caims 1982: 294, Fig. 131c-d.Fenner 1993b: 1105-1106, Lám. 1: Fig. b.

Isophyllastrea rigida (Dana, 1848)

Isophyllastrea rigida.- Squires 1958: 258, Lám. 41: Fig. 3.- Duarte Bello 1961: 42, Figs. 31-32.- Almy y Carrión-Torres 1963: 158, Lám. 18: Fig. b.- Roos 1971: 80-81, Lám. 47: Figs. a-b.- Smith 1971: 92-93, Lám. 43.- Chassaing et al. 1978: 77, Fig. 61.- Castañares y Soto 1982: 317-318, Lám. 20: Figs. a-b.

Mycetophyllia aliciae Wells, 1973

Mycetophyllia aliciae.- Wells 1973: 4143, Figs. 25-28.- Castañares y Soto 1982: 319, Lám. 21: Figs. c-d.- Cairns 1982: 296, Fig. 132f, 133a-b.

\section{Mycetophyllia danaana Milne Edwards \& Haime, 1849}

Mycetophyllia danaana.- Wells 1973: 38 (clave de identificación), Figs. 17-18.- Castañares y Soto 1982: 318-319, Lám. 21: Figs. ab.- Cairns 1982: 296, 299, Fig. 132a-c.
Mycetophyllia ferox Wells, 1973

Mycetophyllia lamarckana.- Roos 1971:

82, Láms. 49, 51.

Mycetophyllia lamarckiana.- Smith 1971: 93-94, Lám. 45.

Mycetophyllia ferox.- Wells 1973: 40-41, Figs. 22-24.- Castañares y Soto 1982: 319, Lám. 22: Fig. a.- Cairns 1982: 299, Fig. 133c-d.

\section{Mycetophyllia lamarckiana Milne Edwards \& Haime, 1848}

Mycetophyllia lamarckiana.- Duarte Bello 1961: 58, Figs. 47-48.- Almy y Carrión-Torres 1963: 158-159, Lám. 19: Fig. a.- Smith 1971: 93-94, Lám. 44 ( no-Lám. $45=$ = . ferox). Wells 1973: 38, Fig. 16.- Chassaing et al. 1978: 79, Fig. 64.- Castañares y Soto 1982: 318, Lám. 20: Figs. c-d.- Cairns 1982: 296, Fig. 132e.

No Mycetophyllia lamarckiana.- Roos 1971: 82, Láms. 49 y 51(= M. ferox $)$.

Mycetophyllia ressi Wells, 1973

Mycetophyllia ressi.- Wells 1973: 36, 39, Figs. 19, 20a-b, 21.

Observaciones: Según J. Lang (Wells 1973), esta es la única especie del Caribe en la que los pólipos carecen de tentáculos.

\section{Familia Faviidae \\ Manicina areolata (Linnaeus, 1758)}

Manicina areolata.- Squires 1958: 254255, Lám. 37: Figs. 1-3.- Duarte Bello 1961: 48, Figs. 37-38.- Almy y Carrión-Torres 1963: 152-153, Lám. 11: Fig. b.- Olivares y Leonard 1971: 59, Lám. 4: Figs. c-d.- Roos 1971: 72, Lám. 35 Fig. b.- Smith 1971: 83, Láms. 2527.- Chassaing et al. 1978: 70, 72 Figs. 41-42.Castañares y Soto 1982: 313, Lám. 13: Figs. ab.- Cairns 1982: 285, 287, Fig. 126e-f.

Manicina mayori.- Smith 1971: 83-84.Cortés y Guzmán 1985: 73, Fig. 24a.

Observaciones: Se propone la existencia de dos formas de crecimiento de esta especie (Wells 1973), Manicina areolata forma areolata y $M$. areolata forma mayori. Se diferencian principalmente por que la forma areolata 
presenta un valle central continuo y un pedúnculo, mientras que la forma mayori presenta valles laterales cortos y una base plana. En el Atlántico mexicano se registran ambas formas.

\section{Favia conferta Verrill, 1868}

Favia conferta.- Smith 1971: 79, Lám. 18.- Reyes-Castro et al. 1989: 4, Figs. 1a, b.

\section{Favia fragum (Esper, 1797)}

Favia fragum.- Squires 1958: 253, Lám. 34: Figs. 2-3.- Duarte Bello 1961: 40, Figs. 29-30.Almy y Carrión-Torres 1963: 150, Lám. 10: Fig. a.- Olivares y Leonard 1971: 56, 59, Lám. 4: Figs. a-b.- Roos 1971: 68-69, Lám. 26 Figs. a-b, Lám. 27, Lám. 28 Fig. a.- Smith 1971: 79-80, Láms. 16-17.- Chassaing et al. 1978: 69, Fig. 32 (a la izquierda).- Castañares y Soto 1982: 311, Lám. 11: Figs. a-b.- Cairns 1982: 283, Fig. 125d.- Cortés y Guzmán 1985: 73, Fig. 23a-b.Reyes-Castro et al. 1989: 4, fig. 1a-b.

Favia gravida Verrill, 1868

Favia gravida.- Almy y Carrión-Torres 1963: 150-151, Lám. 10: Fig. a.- Smith 1971: 80. -Reyes-Castro et al. 1989: 5, Fig. 2.

Colpophyllia breviserialis Milne Edwards \& Haime, 1849

Colpophyllia breviserialis.- Wells 1973: 32-33, Fig. 15.- Cortés y Guzmán 1985: 71, Fig. 18a-b.

Colpophyllia natans (Houttuyn, 1772)

Colpophyllia natans.- Duarte Bello 1961: 26, Figs. 15-16.- Almy y Carrión-Torres 1963: 153, Lám. 12: Fig. b.- Olivares y Leonard 1971: 53, Lám. 2: Figs. a-b.- Roos 1971: 73, Lám. 33 Figs. a-b.- Smith 1971: 82-83, Lám. 23.- Castañares y Soto 1982: 313-314, Lám. 14: Figs. a-b.- Cairns 1982: 287, Fig. 127a-b.Cortés y Guzmán 1985: 71-72, Fig. 19a-b.

Colpophyllia amaranthus.- Smith 1971: 82, Lám. 24.

Diploria clivosa (Ellis \& Solander, 1786)

Diploria clivosa.- Squires 1958: 253, Lám. 42: Fig. 2.- Duarte Bello 1961: 32, Figs.
21-22.- Almy y Carrión-Torres 1963: 151, Lám. 10: Fig. b.- Olivares y Leonard 1971: 56, Lám. 3: Figs. a-b.- Roos 1971: 69-70, Lám. 28 Fig. b (no-Lám. 23 = Montastraea cavernosa), Lám. 30, Lám. 31.- Smith 1971: 80-81, Lám. 19, Lám. 20.- Chassaing et al. 1978: 69-70, Figs. 38-39 (no D. strigosa).- Castañares y Soto 1982: 311-312, Lám. 11: Figs. c-d.- Cairns 1982: 285, Fig. 125e-f.- Cortés y Guzmán 1985: 72, Fig. 20a-b.

Diploria labyrinthiformis (Linnaeus, 1758)

Diploria labyrinthiformis.- Duarte-Bello 1961: 36, Figs. 25-26.- Almy y Carrión-Torres 1963: 151, Lám. 11: Fig. a.- Roos 1971: 71, Lám. 32 Figs. a-b.- Smith 1971: 81, Lám. 21.Chassaing et al. 1978: 70, Figs. 36-37.- Castañares y Soto 1982: 312-313, Lám. 12: Figs. cd (no-Lám. 2 = Acropora palmata).- Cairns 1982: 285, Fig. 126c-d.- Cortés y Guzmán 1985: 72, Fig. 21a-b.

Diploria strigosa (Ellis \& Solander, 1786)

Diploria strigosa.- Squires 1958: 253254, Lám. 42: Fig. 1.- Duarte Bello 1961: 34, Figs. 23-24.- Almy y Carrión-Torres 1963: 151-152, Lám. 10: Fig. b.- Olivares y Leonard 1971: 56, Lám. 3: Figs. c-d.- Roos 1971: 7071, Lám. 29 Figs. a-b.- Smith 1971: 81-82, Lám. 22.- Chassaing et al. 1978: 70, Figs. 3435 (no Figs. 38-39).- Castañares y Soto 1982: 312, Lám. 12: Figs. a-b.- Cairns 1982: 285, Fig. 126a-b.- Cortés y Guzmán 1985: 72-73, Fig. 22a-b.

\section{Montastraea annularis (Ellis \& Solander,} 1786)

Montastrea annularis.- Squires 1958: 256, Lám. 40: Fig. 3.- Duarte Bello 1961: 54, Figs. 43-44.- Almy y Carrión-Torres 1963: 151-152, Lám. 10: Fig. b.- Olivares y Leonard 1971: 61, Lám. 6: Fig. d.- Roos 1971: 65-66, Lám. 24 Fig. b, Lám. 25 Fig. b.- Smith 1971: 85-86 (no Láms. 31-32 = M. faveolata).- Chassaing et al. 1978: 72, Fig. 45.- Castañares y Soto 1982: 314, Lám. 15: Fig. a.Cairns 1982: 287, Fig. 127c-d.- Cortés y Guzmán 1985: 74, Fig. 25a-b. 
Montastraea annularis.- Knowlton et al. 1992: Fig. 1a ("morphotype 1").- Weil y Knowlton 1994: 155, 158-159, Figs. 3a, 4a-b, 5a, 7a-b.

Montastraea cavernosa (Linnaeus, 1767)

Montastrea cavernosa.- Squires 1958: 255-256, Lám. 40: Figs. 1-2.- Duarte Bello 1961: 52, Figs. 41-42.- Almy y Carrión-Torres 1963: 154, Lám. 14: Fig. b1-2.- Olivares y Leonard 1971: 61, Lám. 7: Figs. a-b.- Roos 1971: 66-67, Lám. 22 Fig. c, Lám. 23.- Smith 1971: 86-87, Lám. 33.- Chassaing et al. 1978: 74, Fig. 46.- Castañares y Soto 1982: 314-315, Lám. 15: Figs. b-c.- Cairns 1982: 287, Fig. 127e-f.Cortés y Guzmán 1985: 74-75, Figs. 26-27.

Montastrea braziliana.- Smith 1971: 86.

\section{Montastraea faveolata (Ellis \& Solander, 1786)}

Montastrea annularis.- Almy y CarriónTorres 1963: 151-152.- Roos 1971: 65-66.Smith 1971: 85-86, Láms. 31-32.- Castañares y Soto 1982: 314.- Cairns 1982: 287.

Montastraea annularis.- Knowlton et al. 1992: Fig. 1b ("morphotype 2").

Montastraea faveolata.- Weil y Knowlton 1994: 160-162, Figs. 3b, 4c-d, 5b, d-f izquierda y derecha, 7c-d.

Montastraea franksi (Gregory, 1895)

Montastrea annularis.- Almy y CarriónTorres 1963: 151-152.- Smith 1971: 85-86.Castañares y Soto 1982: 314.- Cairns 1982: 287. Montastraea annularis.- Knowlton et al. 1992: Fig. 1c ("morphotype 3").

Montastraea franksi.- Weil y Knowlton 1994: 162-164, Figs. 3c, 4e-f, 5c-f-centro, 7e.

Cladocora arbuscula (Lesueur, 1821)

Cladocora arbuscula.- Duarte Bello 1961:
24, Figs. 13-14.- Almy y Carrión-Torres 1963 : 153-154, Lám. 13: Fig. a.- Roos 1971: 65, Lám. 22 Fig. a-b.- Smith 1971: 84, Lám. 28. Chassaing et al. 1978: 72, Fig. 44.- Cairns 1982: 287, Fig. 128a.- Cortés y Guzmán 1985: 74, Fig. 24b.- Reyes-Castro et al. 1989: 5-6, Fig. 3.

Solenastrea bournoni Milne Edwards \& Haime, 1849

Solenastrea bournoni.- Duarte Bello 1961: 76, Figs. 65-66.- Almy y Carrión-Torres 1963: 154, Lám. 13: Fig. b.- Roos 1971: 67-68, Lám. 24 Fig. a, Lám. 25a.- Smith 1971: 8485.- Castañares y Soto 1982: 315, Lám. 16: Figs. a-b (no-Lám. 6 = Agaricia tenuifolia).

Solenastrea hyades (Dana, 1846)

Solenastrea hyades.- Duarte Bello 1961: 78, Figs. 67-68.- Smith 1971: 85, Láms. 29-30.

Subordo Caryophyllina Familia Caryophylliidae

Eusmilia fastigiata Gray, 1847

Eusmilia fastigiata.- Duarte Bello 1961: 38, Figs. 27-28.- Almy y Carrión-Torres 1963: 160-161, Lám. 21: Fig. a.- Roos 1971: 83, Lám. 52 Figs. a-b.- Smith 1971: 95, Lám. 48.Wells 1973: 47-48, Figs. 34a-b, 35 derecha e izquierda.- Chassaing et al. 1978: 79, Figs. 6566.- Castañares y Soto 1982: 319-320, Lám. 22: Figs. c-d.- Cairns 1982: 299, Fig. 133e-f.

Observaciones: Se distinguen 2 formas diferentes de esta especie, la forma fastigiata y la forma flabellata que se distingue de la primera por tener series policéntricas mas largas que dan lugar a ramas flabeladas; la segunda forma, únicamente se ha registrado en Cozumel, Q. Roo (Fenner 1993a).

Clave para la identificación de las especies

1a. Corales sin coralitos ni valles, con la superficie perforada con pequeños poros ................................................. 2

lb. Corales con coralitos con escleroseptos radiales o con valles con escleroseptos paralelos .................................... 4

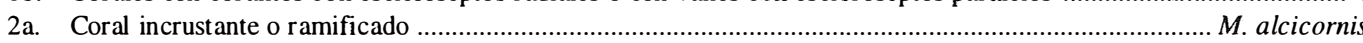

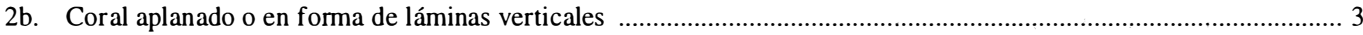

3a. Coral en forma de láminas verticales ............................................................................................ M. complanata

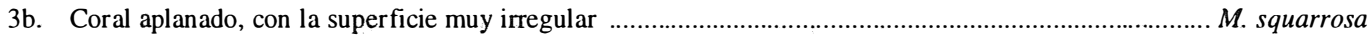

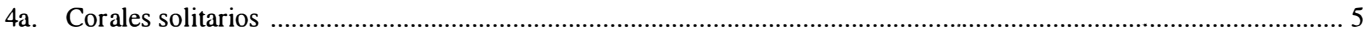

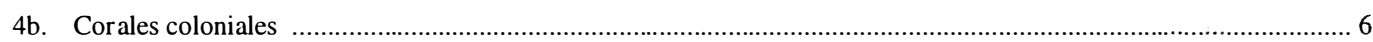


5a. Escleroseptos toscamente dentados con menos de 5 dientes triangulares por $\mathrm{cm}$......................................... S. lacera

5b. Escleroseptos finamente dentados más de 5 dientes largos y delgados por $\mathrm{cm}$........................................... S. cubensis

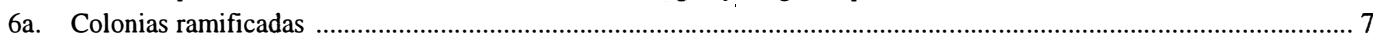

6b. Colonias incrustantes, submasivas, masivas o en forma de láminas u hojas, pero nunca ramificadas ......................19

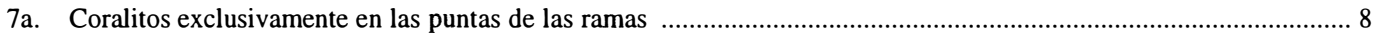

7b. Coralitos sobre toda la superficie de las ramas ................................................................................................ 10

8a. Copas pequeñas, de $3-4 \mathrm{~mm}$ de diámetro .............................................................................................. C. arbuscula

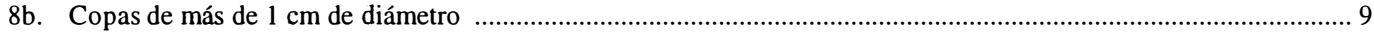

9a. Copas ovales con escleroseptos de bordes lisos ................................................................................. E. fastigiata

9b. Copas circulares con escleroseptos dentados ......................................................................................... M. angulosa

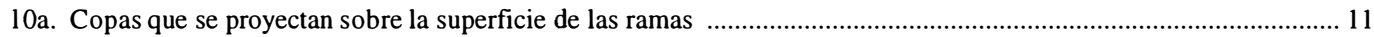

10b. Copas que no se proyectan sobre la superficie de las ramas ........................................................................... 17

11a. Copas en forma de proyecciones cilíndricas de paredes perforadas ................................................................. 12

11b. Copas en el ápice de proyecciones cónicas bajas de paredes sólidas ..................................................................14

12a. Ramas aplanadas a manera de abanico .................................................................................................. A.. palmata

12b. Ramas cilíndricas divergentes, colonias de apariencia arbustiva .................................................................. 13

13a. Ramas largas, rara vez fusionadas, colonias poco tupidas ................................................................... A. cervicornis

13b. Ramas fusionadas, colonias tupidas ................................................................................................. A. prolifera

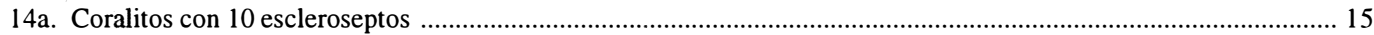

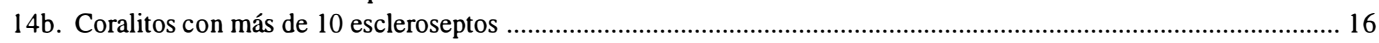

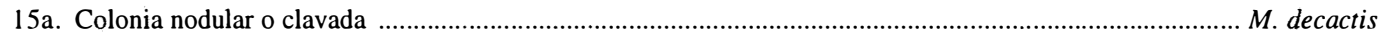

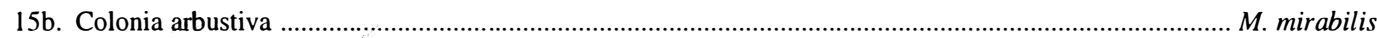

16a. Ramas cortas fusionadas de menos de $10 \mathrm{~mm}$ de ancho ............................................................................. O. diffusa

16b. Ramas largas y torcidas de hasta $2 \mathrm{~cm}$ de diámetro ........................................................................ O. valenciennesi

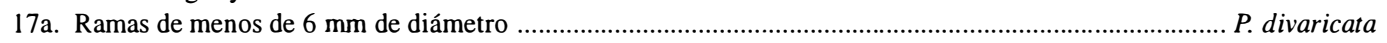

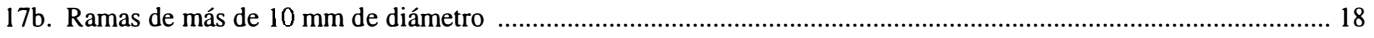

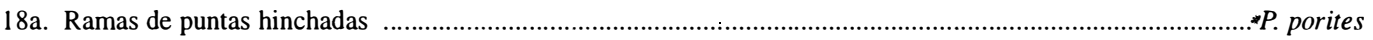

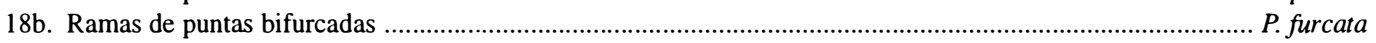

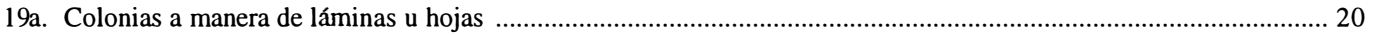

19b. Colonias incrustantes, masivas o submasivas, pero nunca en forma de hoja .......................................................... 30

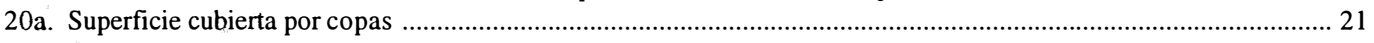

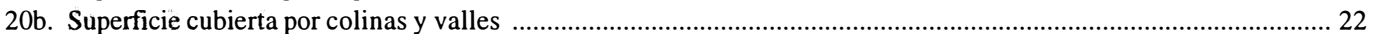

21a. Coralitos cerioides con 12 escleroseptos ......................................................................................... P. colonensis

$2 \mathrm{lb}$. Coralitos con un aspecto marcadamente protuberante con 10 a 15 escleroseptos ...................................... M. ressi

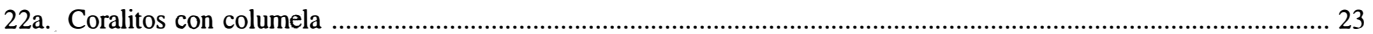

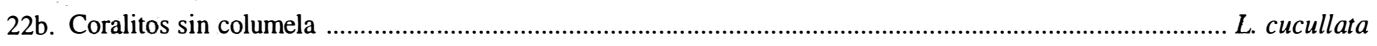

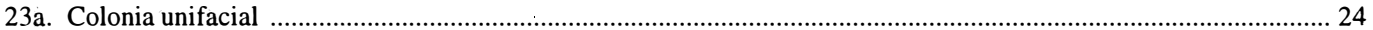

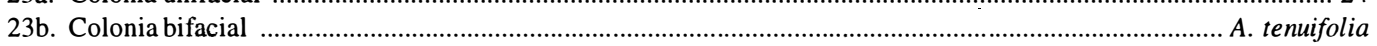

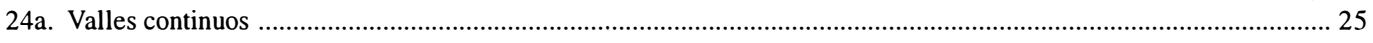

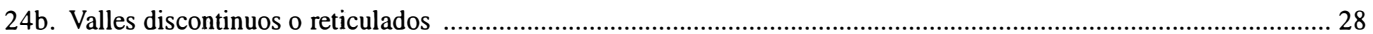

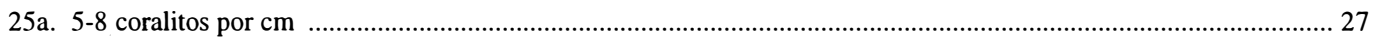

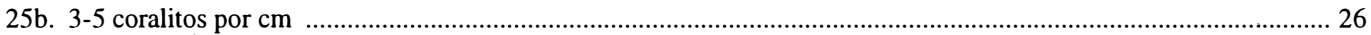

26a. Septos alternados más delgados que los interespacios ........................................................................... A. lamarcki

26b. Septos iguales o casi iguales a los interespacios .................................................................................. A. grahamae

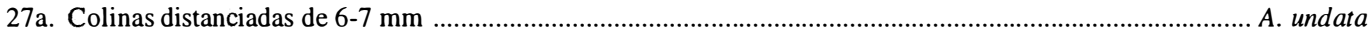

27b. Colinas distanciadas de $2-4 \mathrm{~mm}$.......................................................................................................... A. fragilis

28. Superficie de la colonia sin proyecciones erectas bifaciales ...................................................... agaricites purpurea

28b. Superficie de la colonia con proyecciones erectas bifaciales ….................................................................... 29

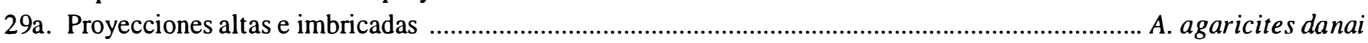

29b. Proyecciones bajas y gruesas, en forma de carinas .............................................................. A. agaricites carinata

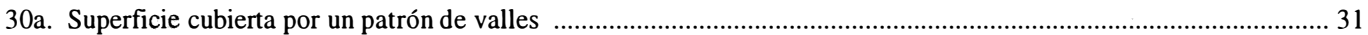

30b. Superficie cubierta por copas circulares, ovaladas o elongadas que no se unen formando valles ............................48

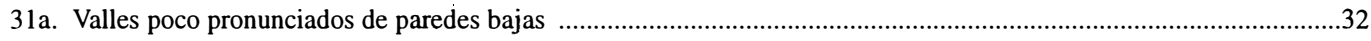

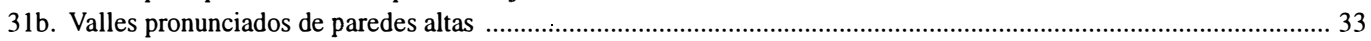

32a. Colonias incrustantes cóncavas, $4-5$ coralitos por $\mathrm{cm}$, separados $3.5-4 \mathrm{~mm}$ radialmente ........... agaricites agaricites

32b. Colonias submasivas convexas, $5-7$ coralitos por $\mathrm{cm}$, separados $2 \mathrm{~mm}$ radialmente ...................... agaricites humilis

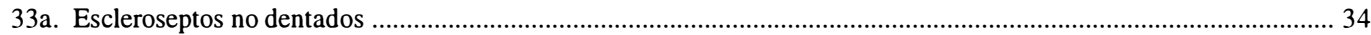

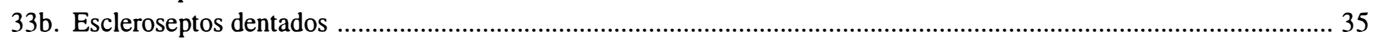


34a. Pilares rectos y gruesos, algunas veces muy grandes

D. cylindrus

34b. Colonias masivas, hemisféricas, nunca forman columnas o pilares

M. meandrites

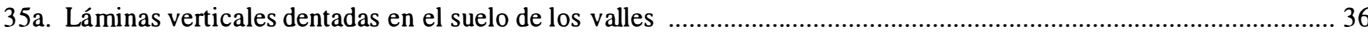

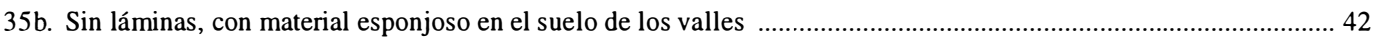

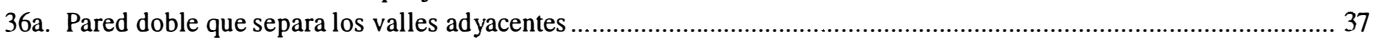

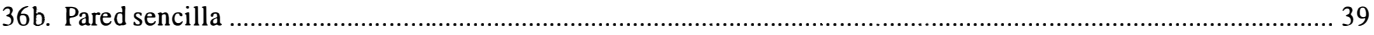

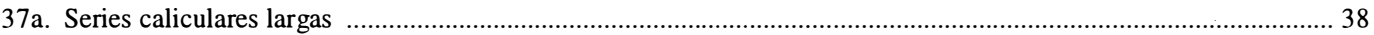

37b. Series caliculares cortas, rara vez con más de un centro .............................................................. C. breviserialis

38a. Superficie inferior de la colonia con un pedúnculo ancho, 11 escleroseptos por $\mathrm{cm}$.................. natans amaranthus

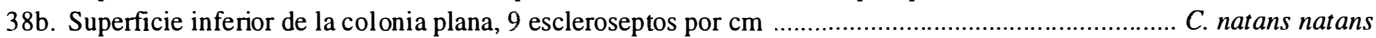

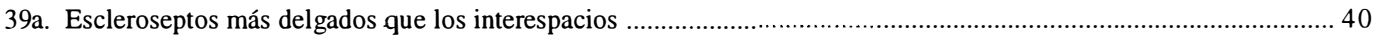

39b. Escleroseptos iguales o más gruesos que los interespacios ........................................................................ M. aliciae

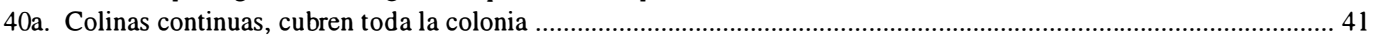

40b. Colinas radiales, ausentes en el centro .................................................................................. M. lamarckiana

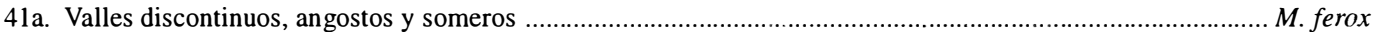

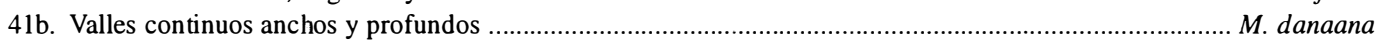

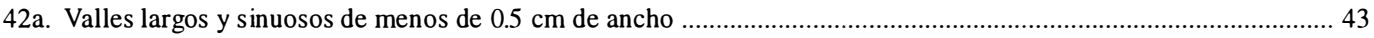

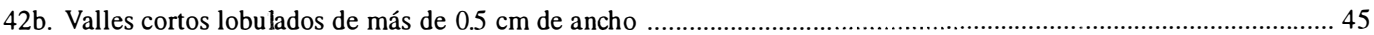

43a. Mas de 20 escleroseptos por $\mathrm{cm}$. Paredes entre valles con crestas afiladas sin surco ................................ D. clivosa

43b. Menos de 20 escleroseptos por $\mathrm{cm}$. Paredes redondeadas o aplanadas, algunas veces con un surco .......................44

44a. Paredes con un surco en la parte superior .............................................................................. D. labyrinthiformis

44b. Paredes sin surco, salvo en algunas raras ocasiones que se presenta en las orillas de la colonia ................. strigosa

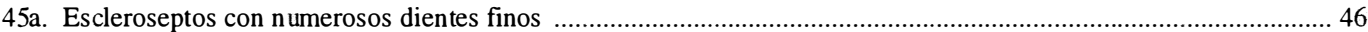

45b. Escleroseptos con pocos dientes toscos prominentes .................................................................................... 47

46a. Superficie cubierta por un solo valle central continuo y varios valles laterales .................... areolata forma areolata

46b. Superficie cubierta por muchos valles discontinuos que atraviesan la colonia transversalmente

47. Valles de $25 \mathrm{~cm}$.

47b. Valles de $1.5 \mathrm{~cm}$ de ancho, 12 escleroseptos por $\mathrm{cm}$.......................................................................... I. multiflora

48. Diámetro de las copas mayor de $1 \mathrm{~cm}$.................................................................................................. I. rida

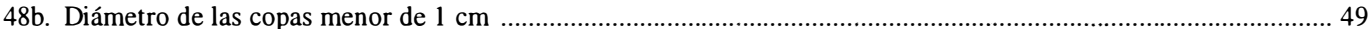

49a. Coral muy poroso, las copas siempre con paredes comunes, nunca separadas ................................................... 50

49b. Coral no poroso, las copas generalmente separadas, ocasionalmente con paredes fusionadas ................................ 53

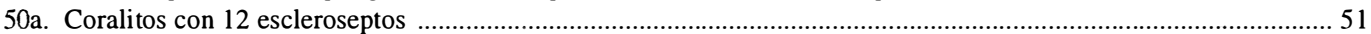

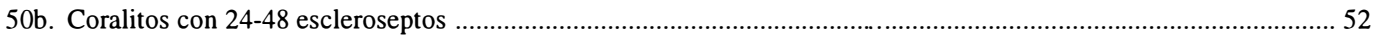

51a. Coralitos someros de 0.7 a $1.2 \mathrm{~mm}$ de diámetro, separados de 0.2 a $0.3 \mathrm{~mm}$..................................... P. branneri

51b. Coralitos profundos de 1.2 a $1.5 \mathrm{~mm}$ de diámetro y separados de 0.5 a $0.8 \mathrm{~mm}$................................. P. astreoides

52a. Coralitos de 1.5 a $4.2 \mathrm{~mm}$ de diámetro. Borde interno de los escleroseptos perpendicular .........................S. radians

52b. Coralitos de 2.6 a $5.0 \mathrm{~mm}$ de diámetro. Borde interno de los escleroseptos cae en una pendiente de $45^{\circ} \ldots$. S. siderea

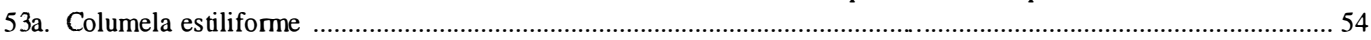

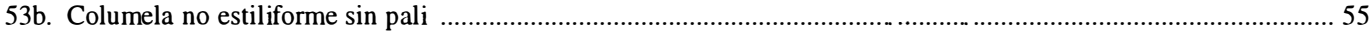

54a. Coralitos de 2.6-3.0 mm de diámetro, con 24 escleroseptos exertos ……............................................ S. intersepta

54b. Coralitos de 1.5-2.5 mm de diámetro con 10 escleroseptos cada uno (forma incrustante) .......................... decactis

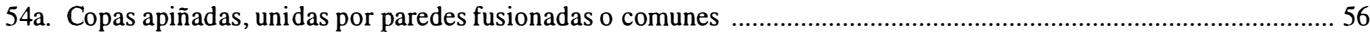

54b. Copas más o menos apiñadas, paredes siempre separadas ............................................................................ 57

56a. Copas de 4.5-6.5 mm de diámetro, circulares u ovales, lobulares o poligonales cuando el coral esta maduro, elongadas o unidas en series

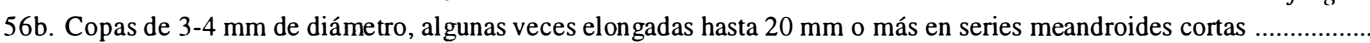

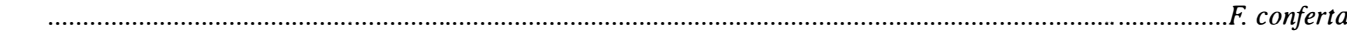

57a. Copas menores a $6 \mathrm{~mm}$ de ancho, pero pueden estar elongadas hasta $30 \mathrm{~mm}$ de largo ......................................5

57b. Copas siempre circulares, de al menos $6 \mathrm{~mm}$ de diámetro, cenostelo no granular .................................................. caverno

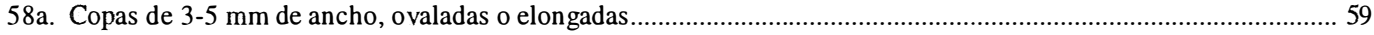

58b. Copas de 2-3.5 mm de diámetro, circulares o poligonales, nunca elongadas ............................................................. 60

59a. Escleroseptos dentados de lados espinosos. Copas de hasta $8 \mathrm{~mm}$ de largo ............................................... F. gravida

59b. Escleroseptos aserrados. Copas ovaladas de 3 a $9 \mathrm{~mm}$ de largo, cenostelo granular .................................. D. stokesi

60a. Coral itos de 3-3.5 mm de diámetro, escleroseptos no exertos ................................................................... S. hyades

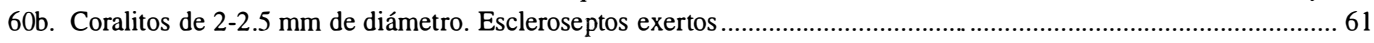

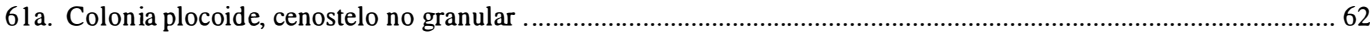


61b. Coral no plocoide, cenostelo granular..... S. bournoni

62a. Superficie de la colonia lisa con coralitos uniformemente distribuidos ................................................................63

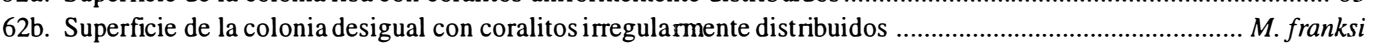

63a. Coral columnar, el tejido vivo se restringe a la parte superior de la columna ............................................. annularis 63b. Coral crustoso, hemisférico o masivo, alto, con proyecciones laterales en declive ................................... faveolata

\section{AGRADECIMIENTOS}

Agradecemos los comentarios hechos al manuscrito original por tres revisores anónimos, S.I. Salazar-Vallejo y M. Elías-Gutiérrez (ECOSUR). El manuscrito se vio enriquecido con los comentarios e información que D.P. Fenner (AIMS) nos proporcionó. Este trabajo fue financiado por el proyecto Arrecifes Coralinos de ECOSUR y por fondos de CONACyT al proyecto 4120P-N9607.

\section{RESUMEN}

Se presenta una lista anotada y revisada de cincuenta y siete especies de corales pétreos (tres mileporinos y cincuenta y cuatro escleractinios) del litoral oriental mexicano. En este informe se incluyen sinonimias y una clave simplificada para las especies de la lista. En aquellos casos en que los diferentes autores no se ponen de acuerdo en la posición taxonómica y/o la identificación de las especies se hacen observaciones morfológicas y taxonómicas, así como cuando existe información taxonómica pertinente, o cuando los datos de distribución son relevantes para algunas especies en el Atlántico mexicano. También, se presenta un análisis cualitativo sobre la abundancia y distribución geográfica de las especies, y datos sobre su ámbito batimétrico. Existe una reducción en el número de géneros y especies del Caribe mexicano hacia el Golfo de México y, dentro de este, del Banco de Campeche hacia Veracruz. Aún más, el número de especies abundantes es menor en el Golfo de México que en el Caribe mexicano y las especies comunes en todas las zonas arrecifales presentan un ámbito batimétrico más amplio en el Caribe mexicano.

\section{REFERENCIAS}

Almy C. \& C. Carrión-Torres. 1963. Shallow-water stony corals of Puerto Rico. Caribb. J. Sci. 3: 133-162.

Boschma, H. 1956. Milleporina and Stylasterina, p. F90F106. In R.C. Moore (ed.). Treatise on invertebrate paleontology. Geol. Soc. Amer. and Univ. Kansas, Lawrence, Kansas.
Cairns, S.D. 1982. Stony corals (Cnidaria, Hydrozoa, Scleractinia) of Carrie Bow Cay, Belize. Smith. Contr. Mar. Sci. 12: 271-302.

Carricart-Ganivet, J.P. \& A.U. Beltrán-Torres. 1997. Lista de corales pétreos (Hydrozoa: Milleporina, Stylasterina; Anthozoa: Scleractinia) de aguas someras del Banco de Campeche, México. Rev. Biol. Trop. 44/45: 619-622.

Carricart-Ganivet, J.P. \& G. Horta-Puga. 1990. Some stony corals (Scleractinia, Milleporidae) of Majahual, Quintana Roo, Mexico. Rev. Zool., ENEP Iztacala, UNAM 2: 3-13.

Carricart-Ganivet, J.P. \& G. Horta-Puga. 1993. Arrecifes de coral en México, p 81-92. In S.I. Salazar-Vallejo \& N.E. González (eds.). Biodiversidad marina y costera de México. Comisión Nacional para el Conocimiento y Uso de la Biodiversidad y Centro de Investigaciones de Quintana Roo, México.

Castañares, L.G. \& L.A. Soto. 1982. Estudios sobre los corales escleractinios hermatípicos de la costa noreste de la Península de Yucatán, México. Parte I: Sinopsis taxonómica de 38 especies (Cnidaria. Anthozoa, Scleractinia). An. Inst. Cienc. Mar Limnol. UNAM 9: 295-344.

Chassaing, J.P., A. Delplanque \& J. Laborel. 1978. Coraux des Antilles Françaises. Rev. Franç. Aquar. Herp. 3: 57-83.

Chávez, E.A. \& E. Hidalgo. 1984. Spatial structure of benthic communities of Banco Chinchorro, Mexico. Ads. Reef. Sci. Joint. Meet. ISRS and Atoll Reef Committee. University of Miami, Florida Abs: 19-20.

Cortés, J. \& H. Guzmán. 1985. Organismos de los arrecifes coralinos de Costa Rica. III: Descripción y distribución geográfica de corales escleractinios (Cnidaria: Anthozoa: Scleractinia) de la costa Caribe. Brenesia 24: 63-123.

Duarte-Bello, P.P. 1961. Corales de los arrecifes cubanos. Acuario Nac., Ser. Educ. (Cuba) No. 2: 1-85.

Farrel, T.M., C.F. D'Elia, L. Lubbers III \& L.J. Pastor. 1983. Hermatypic coral diversity and reef zonation at Cayo Arcas, Campeche Gulf of Mexico. Atoll. Res. Bull. 270: 1-13. 
Fenner, D.P. 1988. Some leeward reefs and corals of Cozumel, Mexico. Bull. Mar. Sci. 42: 133-144.

Fenner, D.H. 1993a. Some reefs and corals of Roatan (Honduras), Cayman Brac, and Little Cayman. Atoll Res. Bull. 388: 1-30.

Fenner, D.P. 1993b. Species distinctions among several Caribbean stony corals. Bull. Mar. Sci. 53: 1099 1116.

Fenner, D.P. 1999. New observations on the stony coral (Scleractinia, Milleporidae, and Stylasteridae) species of Belize (Central America) and Cozumel (Mexico). Bull. Mar. Sci. 64: in .

Heilprin, A. 1890. The Corals and coral reefs of the western waters of the Gulf of Mexico. Proc. Acad. Nat. Sci. Phil. 42: 303-316.

Horta-Puga G. \& J.P. Carricart-Ganivet. 1993. Corales pétreos recientes (Milleporina, Stylasterina y Scleractinia) de México. p 66-80. In S.I. Salazar-Vallejo \& N.E. González (eds.). Biodiversidad Marina y Costera de México. Comisión Nacional para el Conocimiento y Uso de la Biodiversidad y Centro de Investigaciones de Quintana Roo, México.

Jordán, E. 1979. Estructura y composición de arrecifes coralinos en la región noreste de la Península de Yucatán, México. An. Inst. Cienc. Mar Limnol. UNAM 6: 69-86.

Jordán, E. 1980. Arrecifes coralinos del noreste del a Península de Yucatán: Estructura comunitaria, un estimador del desarrollo arrecifal. Tesis Doctoral, Instituto de Ciencias del Mar y Limnología, Universidad Autónoma de México, 117 p.

Jordán, E. 1988. Arrecifes profundos en la Isla de Cozumel, México. An. Inst. Cienc. Mar Limnol. UNAM 15: 195-208.

Jordán, E. 1990. Corales escleractinios y gorgonáceos del ambiente arrecifal de Sian Ka'an. p 127-130. In D. Navarro \& J.G. Robinson (eds.). Diversidad biológica en la Reserva de la Biosfera de Sian Ka'an, Quintana Roo, México. Centro de Investigaciones de Quintana Roo, México.

Jordán, E. 1993. El ecosistema arrecifal coralino del Atlántico mexicano. Rev. Soc. Mex. Hist. Nat Vol. Esp. 46: $157-175$.

Jordán, E. \& E. Martin. 1987. Chinchorro: morphology and composition of a Caribbean Atoll. Atoll Res. Bull. 310: 1-27.
Knowlton, N.E., E. Weil, L.A. Weigth \& H. Guzmán 1992. Sibiling species in Montastraea annularis, coral bleaching, and the coral climate record. Science 255: $330-333$

Kornicker, L.S., F. Bonet, R. Cann \& C.M. Hoskin. 1959. Alacran reef, Campeche Bank, Mexico. Pub. Inst Mar. Sci. Univ. Texas 6: 1-22.

Lara, M., C. Padilla, C. García \& J.J. Espejel. 1992. Coral reefs of Veracruz Mexico I. Zonation and community. Proc. $7^{\text {th }}$ Int.Coral Reef Symp. 1: 535-544.

Moore. D.R. 1958. Notes on Blanquilla reef the most northely coral formation in the western Gulf of Mexico. Pub. Inst. Mar. Sci. Univ. Texas: 151-155.

Muckelbauer, G. 1990. The shelf of Cozumel, Mexico: Topography and organisms. Facies 23: 185-240.

Olivares, M.A \& A.B. Leonard. 1971. Algunos corales pétreos de la Bahía de Mochima. Venezuela. Bol. Inst. Oceanogr. Univ. Oriente 10: 49-70.

Padilla, C., D. Gutiérrez, M. Lara \& C. García. 1992. Coral reefs of the biosphere reserve of Sian Ka'an, Quintana Roo, Mexico. Proc. $7^{\text {th }}$ Int.Coral Reef Symp. 2: 986-992.

Reyes-Castro, J., R. Saenz-Morales \& G. Horta-Puga. 1989. Corales pétreos de Isla Contoy y Arrecife Cabezo, Q. Roo, México. Rev. Zool., ENEP Iztacala, UNAM 1: $1-9$

Roos, P.J. 1971. The shallow-water stony corals of the Netherlands Antilles. Stud. Fauna. Curacao and other Caribbean Islands 130: 108 p.

Smith, W. 1971. Atlantic reef corals. University of Miami, Coral Gables, Florida, 164 p.

Squires, D.F. 1958. Stony corals from the vicinity of Bimini, Bahamas, British West Indies. Bull. Am. Mus. Nat. Hist. 115: 221-277.

Tunnell, J.W. 1988. Regional comparison of the southwestern Gulf of Mexico to Caribbean Sea coral reefs. Proc. $6^{\text {th }}$ Int. Coral Reef Symp. Australia, 3: 303308.

Tunnell, J.W., A.A. Rodriguez, R.L. Lehman \& C.R. Beaver. 1993. An ecological characterization of the southern Quintana Roo coral reef system. Center for Coastal Studies, Texas A\&M University-Corpus Christi, Texas. $161 \mathrm{p}$. 
Veron, J.E.N. 1995. Corals in space and time. The biogeography and evolution of the Scleractinia. Cornell University Nueva York. 321 p.

Villalobos, A. 1971. Estudios ecológicos en un arrecife coralino en Veracruz, p. 532-545. Symposium on investigations and resources of the Caribbean Sea and adjacent regions; UNESCO-FAO.

Weerdt, W.H. de. 1984. Taxonomic characters in Caribbean Millepora species (Hydrozoa, Coelenterata). Bijdr. Dierk. 54: 243-262.

Weerdt, W.H. de. 1990. Discontinuous distribution of the tropical West Atlantic Hydrocoral Millepora squarrosa. Beaufortia 41: 195-203.

Weil, E. 1992. Genetic and morphological variation in Caribbean and eastern Pacific Porites (Anthozoa, Scleractinia). Preliminary results. Proc. $7^{\text {th }}$ Int. Coral Reef Symp. Guam, 2: 643-656.
Weil, E. \& N.E. Knowlton. 1994. A multicharacter analysis of the Caribbean coral Montastraea annularis (Ellis \& Solander, 1786) and it's two sibiling species, M. faveolata (Ellis \& Solander, 1786) y M. franksi (Gregory, 1895). Bull. Mar. Sci. 55: 151-175.

Wells, J.W. 1971. Note on the Scleractinian corals Scolymia lacera and $S$. cubensis in Jamaica. Bull. Mar. Sci. 21: 960-963.

Wells, J.W. 1973. New and old Scleractinian corals from Jamaica. Bull. Mar. Sci. 23: 16-58.

Zlatarski, V.N. 1990. Porites colonensis, new species of stony coral (Anthozoa: Scleractinia) of the Caribbean coast of Panama. Proc. Biol. Soc. Wash. 103: 257-264. 


\section{CUADRO 1}

Abundancia relativa de las especies de corales pétreos de aguas someras del Atlántico mexicano

Especies

Millepora alcicornis

M. complanata

M. squarrosa

Stephanocoenia intersepta

Madracis decactis

M. mirabilis

Acropora cervicornis

A. palmata

A. prolifera

Porites astreoides

P. branneri

P. colonensis

$P$. divaricata

$P$. furcata

$P$. porites

Siderastrea radians

$S$. siderea

Agaricia agaricites

A. fragilis

A. grahamae

A. lamarcki

A. tenuifolia

A. undata

Leptoseris cucullata

Oculina difussa

$O$. valenciennesi

Meandrina meandrites

Dichocoenia stokesi

Dendrogyra cylindrus

Scolymia cubensis

S. lacera

Mussa angulosa

Isophyllia multiflora

I. sinuosa

Isophyllastrea rigida

Mycetophyllia aliciae

M. danaana

M. ferox

M. lamarckiana

M. ressi

Manicina areolata

Favia conferta

F. fragum

F. gravida

Colpophyllia breviserialis

C. natans

Diploria clivosa

D. labyrinthiformis

D. strigosa

Montastraea annularis

$M$. cavernosa

$M$. faveolata

M. franksi

Cladocora arbuscula

Solenastrea bournoni

S. hyades

Eusmilia fastigiata

$\mathbf{A}=$ abundante, $\mathbf{C}=$ común, $\mathbf{O}=$ ocasional, $\mathbf{R}=$ raro,$-=$ no registrado

Abundancia relativa

Banco de Campeche

A

A

R

C

C

A

A-C

$\mathrm{O}$

A

-

A-C

C

C-O

C

A-C

O-R

O

Caribe 
Distribución geográfica y batimétrica de las especies de corales pétreos de aguas someras del Atiántico mexicano

Distribución / profundidades en metros

Especies

Millepora alcicornis

M. complanata

M. squarrosa

Stephanocoenia intersepta

Madracis decactis

M. mirabilis

Acropora cervicornis

A. palmata

A. prolifera

Porites astreoides

P. branneri

P. colonensis

P. divaricata

P. furcata

P. porites

Siderastrea radians

S. siderea

Agaricia agaricites

A. fragilis

A. grahamae

A. lamarcki

A. tenuifolia

A. undata

Leptoseris cucullata

Oculina difussa

$O$. valenciennes

Meandrina meandrites

Dichocoenia stokesi

Dendrogyra cylindrus
Veracruz

1

$1-7 \quad 1-7$

$5-6 . \quad 3-21$

$5-6 \quad 1-15$

* $\quad 1-15$

* $\quad 1-15$

$>5 \quad \begin{array}{ll}1-8 \\ 1-24\end{array}$

$25 \quad 3-12$

$\begin{array}{cc} & 1-4 \\ & 1-4 \\ .5-5 & 1-4 \\ 1-15 & 1-20 \\ 2-10 & 1-21 \\ 1-12 & 1-20 \\ 5-15 & 3-24\end{array}$

10-30

$\begin{array}{cc}5-8 & 3-8 \\ * & 7-21\end{array}$

4-18

* 1-3
Banco de Campeche

345

$\begin{array}{llll}1-7 & 1-20 & 1-7 & 1-15\end{array}$

$1-4 \quad 1-13 \quad 1-4 \quad 1-15$

$5-6 \quad 5-30 \quad 5-6 \quad 1-15$

* $1220 \quad 5-6$

$\begin{array}{llll}-10 & 1-15 & 2-8 & 1-8\end{array}$

$\begin{array}{llll}2-6 & 1-12 & * & 1-15\end{array}$

$\begin{array}{cccc}1-7 & 1-7 & 1-7 & 1-7\end{array}$

$\begin{array}{lll}* & 1-30 \quad * \quad>15\end{array}$

$\begin{array}{lll}1-7 & 1-7 & 1-7\end{array}$

$1-5 \quad 1-10 \quad 1->5 \quad>15$

* $\quad 1-15 \quad 1-15 \quad 1-15$

* 7-30 2-10 2-10

$1-12 \quad 1-30 \quad 1-12 \quad 3-15$

$5-15 \quad 1-15$

$0-5$

$$
\text { 8-10 8-10 }
$$

$1-5$ *
Caribe

10

$1-25$

8

$11 \quad 12$

$1-15$

$1-5$

$\begin{array}{lll}1-5 & 1-35 & 1-15\end{array}$

$1-25$
$4-20$
$*$
$1-20$
$1-10$
$1-5$
$1-20$
$1-10$

$1-25$
$1-2$
$1-20$
$1-10$
$1-20$
$1-25$
$1-3$

25
$1-5$
$*$
$10-20$
10

$1-25$
$1-25$
$1-3$

$\begin{array}{ccccc} & 2-50 & & & >15 \\ 1-5 & 3-50 & * & 12-35 & 7-9 \\ & 10-13 & * & 23-35 & 7-15 \\ 1-5 & * & * & 2-28 & 1-11 \\ 1-5 & * & 1-10 & 1-23 & 1-15 \\ & & * & 1 & 1-2 \\ 1-5 & 1-50 & 1-4 & 1-35 & 1-15 \\ & * & & 1 & \\ & * & & & \\ & * & * & 4-20 & 1-9 \\ * & * & * & 2-35 & 7-9 \\ 1-5 & * & * & 1-35 & 1-15 \\ 1-5 & * & * & 1-23 & 6-18 \\ * & 2-50 & 2-27 & 1-35 & 6-18 \\ 1-5 & 5-25 & 6-45 & 1-28 & 1-33 \\ & * & * & 12-35 & 33 \\ & * & & & \\ & * & * & 1-35 & >15 \\ & 5-25 & 1-10 & 1-35 & 1-15 \\ & 25-40 & * & & \\ & 2-90 & * & 18-35 & >15 \\ & & & & \\ & * & * & 1-35 & 2-4 \\ 1-5 & * & * & 1-28 & 5-15 \\ & <10 & * & 1 & 1-15\end{array}$

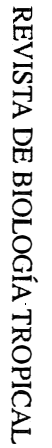




\begin{tabular}{|c|c|c|c|c|c|c|c|c|c|c|c|c|c|}
\hline \multirow[t]{2}{*}{ Especies } & \multicolumn{2}{|c|}{ Veracruz } & \multicolumn{4}{|c|}{ Banco de Campeche } & \multicolumn{7}{|c|}{ Caribe } \\
\hline & 1 & 2 & 3 & 4 & 5 & 6 & 7 & 8 & 9 & 10 & 11 & 12 & 13 \\
\hline Scolymia cubensis & & 6 & & $*$ & & & & & & $*$ & $*$ & $19-35$ & $1-18$ \\
\hline S. lacera & $10-12$ & $3-18$ & & * & & $*$ & 15 & & & $>10$ & $*$ & $19-35$ & $>15$ \\
\hline Mussa angulosa & $10-15$ & $3-15$ & $*$ & $10-15$ & & & $1-3$ & & & $20-30$ & * & $19-35$ & $1-15$ \\
\hline Isophyllia multiflora & & & & & & & - & & $1-5$ & $*$ & & 1 & $1-15$ \\
\hline I. sinuesa & & & & & & & $1-3$ & & $1-5$ & $*$ & $*$ & $2-35$ & $1-15$ \\
\hline Isophyllastrea rigida & & & & & & & $1-10$ & & $1-5$ & $2-30$ & $*$ & $2-35$ & $*$ \\
\hline Mycetophyllia aliciae & & & $12-15$ & $12-15$ & & & $*$ & & & $*$ & $*$ & & $>15$ \\
\hline M. danaana & $>1$ & $>1$ & $>1$ & $1-7$ & $>1$ & $>1$ & $*$ & & & $>50$ & * & $10-35$ & $>8$ \\
\hline M. ferox & $2-12$ & $9-15$ & $2-12$ & $2-17$ & $2-12$ & $2-12$ & $*$ & & & 25 & $*$ & $18-35$ & $>15$ \\
\hline M. lamarckiana & $3-5$ & $3-9$ & $3-5$ & $1-17$ & $3-5$ & $3-5$ & $1-8$ & & & $>20$ & $*$ & $1-35$ & $>33$ \\
\hline M. ressi & & & & & & & & & & $*$ & & & \\
\hline Manicina areolata & & $3-5$ & $*$ & * & & & $1-10$ & $1-5$ & & $2-40$ & $*$ & $1-10$ & $>7$ \\
\hline Favia conferta & & $1-3$ & & & & & & $1-5$ & & & & & \\
\hline F.tragum & & $*$ & $1-2$ & $1-6$ & $1-2$ & $1-2$ & $1-15$ & & $1-5$ & $0-30$ & * & $1-23$ & $1-15$ \\
\hline F. gravida & & & & & & & & & $1-5$ & & & & \\
\hline Colpophyllia breviserialis & & $*$ & & & & & 25 & & & * & * & & \\
\hline C. natans & $2-5$ & $1-40$ & $2-5$ & $10-30$ & $2-5$ & $2-15$ & $1-25$ & & $1-5$ & * & $*$ & $1-35$ & 6 \\
\hline Diploria clivosa & $1-7$ & $1-40$ & $1-13$ & $1-15$ & $1-7$ & $1-15$ & $1-20$ & $1-5$ & $1-5$ & $*$ & $1-27$ & $1-35$ & $1-15$ \\
\hline D. labyrinthiformis & & & $1-5$ & $6-19$ & $1-5$ & $3-15$ & $1-15$ & & & $*$ & $*$ & $1-35$ & $>7$ \\
\hline D. strigosa & $4-10$ & $1-15$ & $1-13$ & $1-15$ & $4-10$ & $1-15$ & $1-20$ & $1-5$ & $1-5$ & $2-40$ & * & $1-35$ & $1-15$ \\
\hline Montastraea annularis & $2-10$ & $3-18$ & $2-18$ & $2-18$ & $2-15$ & $3-18$ & $1-16$ & $1-5$ & & $1-50$ & $1-15$ & $1-15$ & $1-15$ \\
\hline M. cavernosa & $2-6$ & $3-40$ & $2-18$ & $2-27$ & $2-6$ & $1-15$ & $1-25$ & & $1-5$ & $3->50$ & $6-27$ & $1-28$ & $8-33$ \\
\hline M. faveolata & $*$ & $3-22$ & $*$ & $*$ & * & $3-33$ & $*$ & $*$ & & * & $*$ & $2-35$ & $*$ \\
\hline M. franksi & * & $25-40$ & $*$ & $*$ & * & $28-40$ & $*$ & $*$ & & * & $*$ & $28-35$ & $*$ \\
\hline Cladocora arbuscula & & & & & & & & $1-5$ & & & * & & \\
\hline Solenastrea bournoni & & & * & & & & $5-20$ & $1-5$ & & & & $1-35$ & $6-8$ \\
\hline S. hyades & & & & & & & 5 & $1-5$ & & & & & \\
\hline Eusmilia fastigiata & & & $5-15$ & $5-15$ & $5-15$ & $5-15$ & $10-15$ & & & $>25$ & * & $1-35$ & $0-15$ \\
\hline
\end{tabular}

